\title{
A combination of platelet-to-lymphocyte ratio and carbohydrate antigen 19-9 predict early recurrence after resection of pancreatic ductal adenocarcinoma
}

\author{
Shinichi Ikuta ${ }^{1}$, Takashi Sonoda ${ }^{2}$, Tsukasa Aihara $^{1}$, Naoki Yamanaka ${ }^{1}$ \\ ${ }^{1}$ Department of Surgery, ${ }^{2}$ Department of Medical Oncology, Meiwa Hospital, Nishinomiya, Hyogo, Japan \\ Contributions: (I) Conception and design: S Ikuta; (II) Administrative support: N Yamanaka; (III) Provision of study material or patients: All authors; \\ (IV) Collection and assembly of data: S Ikuta; (V) Data analysis and interpretation: S Ikuta; (VI) Manuscript writing: All authors. (VII) Final approval \\ of manuscript: All authors. \\ Correspondence to: Shinichi Ikuta. Department of Surgery, Meiwa Hospital, Agenaruo 4-31, Nishinomiya, Hyogo 663-8186, Japan. \\ Email: g2s1002@gmail.com.
}

Background: Early recurrence (ER) after surgical resection is an important factor that impacts the survival of patients with pancreatic ductal adenocarcinoma (PDA). We examined risk factors for ER after PDA resection.

Methods: One hundred and thirteen PDA patients who underwent R0 or R1 resection were retrospectively analyzed. Thirty-four patients (30.1\%) received neoadjuvant chemotherapy (NAC) for borderline resectable $(B R)(n=13)$ or initially unresectable $(n=21)$ disease. ER was defined as that diagnosed within 6 months after surgery. Receiver operating characteristic analysis was performed for each variable to determine the optimal cutoff value.

Results: ER occurred in 21 patients (18.6\%). In univariate analysis, preoperative platelet-to-lymphocyte ratio $(\mathrm{PLR}) \geq 144$, carbohydrate antigen (CA) $19-9 \geq 162 \mathrm{U} / \mathrm{mL}$, and pathological tumor size $\geq 3 \mathrm{~cm}$ were significantly associated with ER. High PLR and CA19-9 were independent risk factors for ER by multivariate analysis. Area under the curve (AUC) for predicting ER from a combination of PLR and CA19-9 was 0.765 (95\% confidence interval: 0.664-0.866), which increased the AUC compared to that for each risk factor alone. Patients with both risk factors had a significantly worse overall survival than those with one or no risk factors. When 24 patients with BR-PDA were considered, NAC was associated with a reduced likelihood of having risk factors and with a low ER rate.

Conclusions: A combination of PLR and CA19-9 is a useful predictor of ER after macroscopic curative resection for PDA. NAC may reduce the risk of ER in selected patients.

Keywords: Pancreatic cancer; pancreatic ductal adenocarcinoma (PDA); early recurrence (ER); platelet-tolymphocyte ratio (PLR); neoadjuvant chemotherapy (NAC)

Submitted Jul 08, 2019. Accepted for publication Jul 24, 2019.

doi: $10.21037 /$ atm.2019.08.35

View this article at: http://dx.doi.org/10.21037/atm.2019.08.35

\section{Introduction}

Pancreatic cancer is a major threat to public health and is increasing in incidence in many countries. According to the latest cancer statistics, it is the seventh leading cause of cancer death in both men and women worldwide with nearly as many deaths as cases (1). A projection study estimated that pancreatic cancer will increase from the fourth to the second leading cause of cancer deaths in the United States by 2030 (2). The most common subtype of pancreatic cancer is pancreatic ductal adenocarcinoma (PDA), which is responsible for approximately $95 \%$ of all pancreatic cancers. 
Although complete surgical resection is currently the only treatment that provides long-term survival, only $10-20 \%$ of newly diagnosed PDA patients are candidates for surgery (3). Even if curative surgery is performed, $80 \%$ of patients subsequently experience local and/or systemic recurrence (4). In particular, early recurrence (ER) within 6 months after surgery occurs in a significant proportion of patients and predicts a poor prognosis with a median survival around 9-10 months $(5,6)$, which is attributed in most cases to the presence of micrometastases at the time of surgery $(4,5)$.

Despite improvements in surgical techniques and perioperative management, surgical resection for PDA remains challenging and has unresolved postoperative morbidity and mortality. Newer chemotherapeutic regimens, such as gemcitabine plus nab-paclitaxel and FOLFIRINOX (oxaliplatin, irinotecan, and fluorouracil plus leucovorin), have improved outcomes of advanced PDA patients with a median survival of 8.5-11.1 months $(7,8)$. These combination regimens are also preferred for neoadjuvant chemotherapy (NAC) for borderline resectable (BR) or locally advanced PDA (9-12). Patient stratification based on the risk of ER is thus of critical importance for potentially resectable (R) PDA to avoid futile surgery and for neoadjuvant therapy instead of upfront surgery. High preoperative carbohydrate antigen (CA) 19-9 is a representative risk factor of ER (13-18), but its predictive ability is not always sufficient, and no other reliable risk factors have been identified.

Here, we examined predictive risk factors for ER in PDA patients submitted for macroscopic curative pancreatectomy. To evaluate the protective benefit of neoadjuvant therapy against ER, patients who received NAC were also included and analyzed.

\section{Methods}

\section{Patients}

The records of patients with histopathologically confirmed PDA who underwent pancreatic resection at our department between January 2006 and June 2018 were retrospectively reviewed. After exclusion of 20 patients with macroscopic residual disease (i.e., R2 resection) and 5 with lack of important data, a total of 113 patients were recruited. There were 62 men and 51 women with a median age of 68 years (range, $33-86$ years). This study was approved by the institutional ethics review board of Meiwa Hospital and was conducted in accordance with the Declaration of Helsinki.

\section{Neoadjuvant chemo(radio)therapy}

Tumor status at diagnosis was assessed using various imaging techniques including ultrasonography (US), endoscopic US, multidetector row computed tomography (CT), magnetic resonance imaging (MRI), and positron emission tomography (PET). According to the National Comprehensive Cancer Network guidelines (19), the patients were roughly classified as follows: $\mathrm{R}(\mathrm{n}=67)$; BR $(\mathrm{n}=24)$; and unresectable (UR) $(\mathrm{n}=22)$. All 67 R-PDA patients underwent upfront surgery, whereas 13 of the 24 BR-PDA patients and 21 of the 22 UR-PDA patients received NAC using the following modified regimen: gemcitabine $1,000 \mathrm{mg} / \mathrm{m}^{2} / 2 \mathrm{w}$, docetaxel $35 \mathrm{mg} / \mathrm{m}^{2} / 2 \mathrm{w}$, carboplatin $4 \mathrm{mg} / \mathrm{min} / \mathrm{mL}$ [area under the curve (AUC)] $/ 2 \mathrm{w}$ $(20,21)$. Since December 2016, docetaxel and carboplatin were replaced by nab-paclitaxel $\left(125 \mathrm{mg} / \mathrm{m}^{2}\right)$ and oxaliplatin $\left(85 \mathrm{mg} / \mathrm{m}^{2}\right)$, respectively, based on the approval of gemcitabine plus nab-paclitaxel and FOLFIRINOX for advanced PDA [registered in UMIN-CTR (UMIN 000025398)]. NAC was administered in combination with an oral itraconazole, a commonly used antifungal agent that potently inhibits the Hedgehog pathway, P-glycoprotein, and angiogenesis (22-24), at a daily dose of $400 \mathrm{mg}$ from day-2 to 2 of each treatment cycle. One patient who we finally categorized as UR-PDA had incidental oligo-liver metastases combined with a local $\mathrm{R}$ tumor, but underwent upfront pancreatic resection and simultaneous liver metastasectomy. For BR-PDA patients, there was a trend toward increasing use of NAC in more recent years with 10 patients since February 2016, and 3 patients before 2016 . Concurrent radiotherapy was administered during NAC in 4 patients with UR- $(n=3)$ or BR-PDA $(n=1)$ that abutted the superior mesenteric artery, common hepatic artery, or celiac axis since August 2017. The median duration of NAC was 2.3 months (range, 1.3-5.6 months) and 5.1 months (range, 1.7-35.6 months) for BR- and UR-PDA, respectively, and the median duration from discontinuation of NAC to surgery was 4.7 weeks (range, $2.7-10.3$ weeks).

\section{Pancreatic resection}

The operations were conventional pancreatoduodenectomy 
(PD) in 5 patients, pylorus-preserving $\mathrm{PD}$ in 15 , subtotal stomach-preserving $\mathrm{PD}$ in 55, distal pancreatectomy in 33, and total pancreatectomy in 5. Combined vascular resection was performed in 35 patients, portal vein resection in 33 , common hepatic artery resection in 4 , and celiac axis resection in 2 (there were overlaps). All resected specimens were examined histologically according to the 7th TNM classification system (25). Resection curability was classified as follows: R0, no residual tumor; R1, microscopic residual tumor.

\section{Postoperative follow-up evaluation}

After discharge, patients were followed up with regular physical examination and blood work, including complete blood counts, serum chemistry, liver function, and tumor markers. Imaging examinations that included US, CT, MRI, and PET were performed to screen for recurrent or metastatic disease. Frequency of imaging diagnosis was at the discretion of each physician, but at least every 3 months for the first 2 years. ER was defined as imaging evidence of tumor tissue around the surgical site or the appearance of distant metastases within 6 months after surgery.

\section{Data collection}

Clinicopathological data including age, sex, tumor location, resectability status, preoperative tumor markers, preoperative inflammatory biomarkers, such as neutrophilto-lymphocyte ratio (NLR), platelet-to-lymphocyte ratio (PLR), and modified Glasgow prognostic score (mGPS), pathological tumor size, pTNM stage, histologic grade, resection curability, and administration of NAC and postoperative adjuvant chemotherapy were analyzed. The NLR and PLR were defined as the ratio of the absolute peripheral blood neutrophil and lymphocyte counts and the absolute platelet and lymphocyte counts, respectively. The mGPS was calculated from C-reactive protein (CRP) and albumin as follows: patients with both an elevated CRP level $(>0.5 \mathrm{mg} / \mathrm{dL})$ and hypoalbuminemia $(<3.5 \mathrm{~g} / \mathrm{dL})$ were allocated a score of 2 , patients with only one of these biochemical abnormalities were allocated a score of 1 , and patients with neither of these abnormalities were allocated a score of 0 (26). In patients who received NAC, tumor markers and inflammation-based markers were analyzed using values immediately before surgery, not values at the time of diagnosis.

\section{Statistical analysis}

Univariate analysis was performed for categorical variables with the Fisher's exact test. The receiver operating characteristic (ROC) curve was generated to determine the optimal cutoff values of continuous variables for ER prediction. The AUC was used to estimate the predictive ability of each variable. Multivariate analysis was performed using logistic regression to determine independent risk factors. The median follow-up time was estimated using the reverse Kaplan-Meier method (27). Overall survival (OS) was calculated from the date of surgery to that of death or last contact with the patient, and recurrence-free survival (RFS) was calculated from the date of surgery to that of recurrence. The OS and RFS were assessed using the Kaplan-Meier method and compared by the log-rank test. All statistical analyses were performed with R Statistical Software (Foundation for Statistical Computing, Vienna, Austria), and $\mathrm{P}<0.05$ was considered significant.

\section{Results}

\section{Relationships between clinicopathological factors and ER}

The median follow-up time of all patients was 43.4 months [95\% confidence interval (CI), 28.4-50.8 months]. A total of 21 patients $(18.6 \%)$ had developed ER with a median time of 3.7 months and were assigned to the ER group. The remaining 92 patients who either had evidence of recurrence after 6 months from surgery $(n=49)$ or no evidence of recurrence $(n=43)$ were assigned to the nonER (NER) group. The median time to recurrence in the 49 patients with recurrence after 6 months was 11.1 months. According to the ROC curve analysis, the optimal cutoff values of carcinoembryonic antigen (CEA), CA19-9, NLR, PLR, and tumor size were $3.1 \mathrm{ng} / \mathrm{mL}, 162 \mathrm{U} / \mathrm{mL}, 2.4,144$, and $3 \mathrm{~cm}$, respectively. Table 1 shows univariate analysis of factors associated with ER among the 113 study patients. In comparison with patients of the NER group, patients in the ER group were more likely to have high CA19-9 (66.7\% vs. $34.8 \%, \mathrm{P}=0.013)$, high PLR (81.0\% vs. $41.3 \%, \mathrm{P}=0.001)$, and large tumor size $(61.9 \%$ vs. $35.9 \%, \mathrm{P}=0.047)$. However, none of the other factors including CEA, NLR, mGPS, histologic grade, pTNM stage, NAC, or postoperative adjuvant chemotherapy showed significant differences between the groups. The frequency of distant organ recurrence was higher in the ER group than in the NER group, but the difference was not significant. In contrast, 
Table 1 Relationships between early recurrence and clinicopathological factors

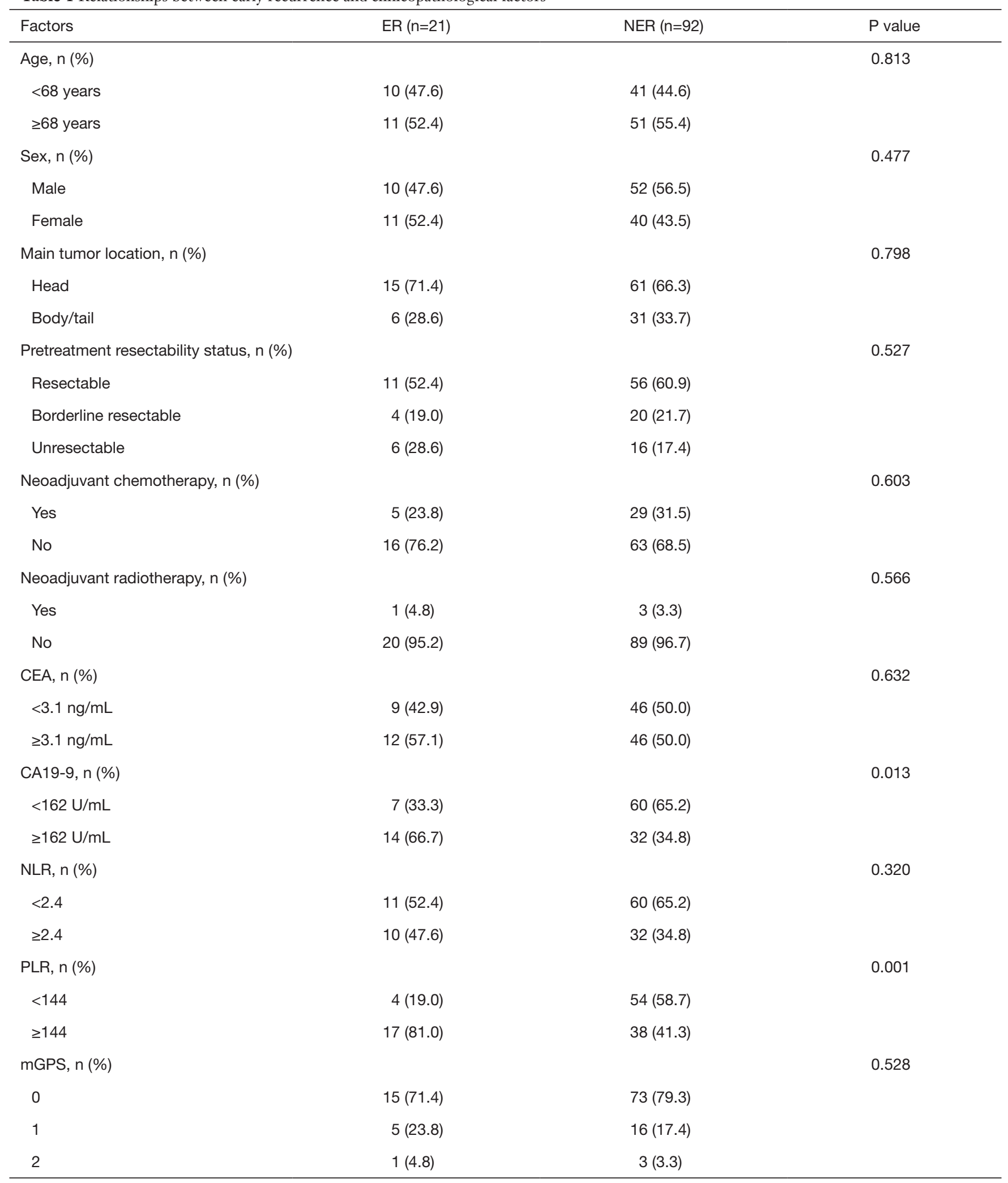

Table 1 (continued) 
Table 1 (continued)

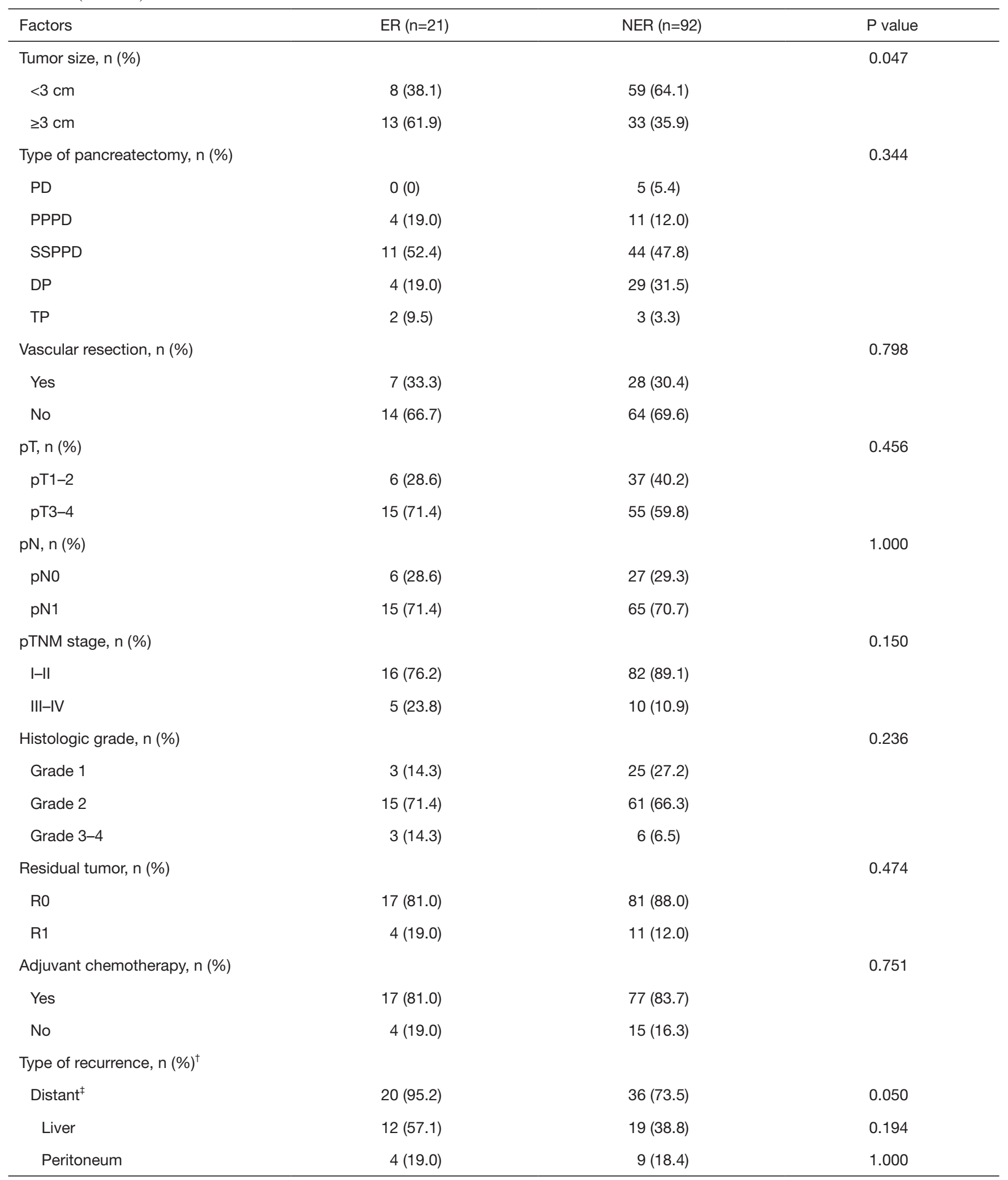

Table 1 (continued) 
Table 1 (continued)

\begin{tabular}{lccc}
\hline Factors & ER $(\mathrm{n}=21)$ & NER $(\mathrm{n}=92)$ & $\mathrm{P}$ value \\
\hline Lungs & $1(4.8)$ & $4(8.2)$ & 1.000 \\
Bone & $2(9.5)$ & $1(2.0)$ & 0.212 \\
Brain & $1(4.8)$ & $0(0)$ & 0.300 \\
Lymph nodes & $2(9.5)$ & $5(10.2)$ & 1.000 \\
Local & $1(4.8)$ & $18(36.7)$ & 0.007 \\
\hline
\end{tabular}

${ }^{\dagger}$, in the NER group, patients with recurrence after 6 months from surgery $(n=49)$ were used as the denominator; ${ }^{\ddagger}$, there were overlapping recurrence sites. ER, early recurrence; NER, non-early recurrence; CEA, carcinoembryonic antigen; CA19-9, carbohydrate antigen 19-9; NLR, neutrophil-to-lymphocyte ratio; PLR, platelet-to-lymphocyte ratio; mGPS, modified Glasgow prognostic score; PD, conventional pancreatoduodenectomy; PPPD, pylorus-preserving pancreatoduodenectomy; SSPPD, subtotal stomach-preserving pancreatoduodenectomy; DP, distal pancreatectomy; TP, total pancreatectomy.

Table 2 Predictive ability of PLR, CA19-9, tumor size, and the combination of PLR and CA19-9 for early recurrence

\begin{tabular}{|c|c|c|c|c|c|c|}
\hline Variable & Cutoff value & AUC $(95 \% \mathrm{Cl})$ & Sensitivity (\%) & Specificity (\%) & PPV (\%) & NPV (\%) \\
\hline CA19-9 & $162 \mathrm{U} / \mathrm{mL}$ & $0.686(0.558-0.814)$ & 66.7 & 65.2 & 30.4 & 89.6 \\
\hline Tumor size & $3.0 \mathrm{~cm}$ & $0.608(0.479-0.737)$ & 61.9 & 64.1 & 28.3 & 88.1 \\
\hline PLR + CA19-9 & - & $0.765(0.664-0.866)$ & 76.2 & 66.3 & 34.0 & 92.4 \\
\hline
\end{tabular}

PLR, platelet-to-lymphocyte ratio; CA19-9, carbohydrate antigen 19-9; AUC, area under the curve; Cl, confidence interval; PPV, positive predictive value; NPV, negative predictive value.

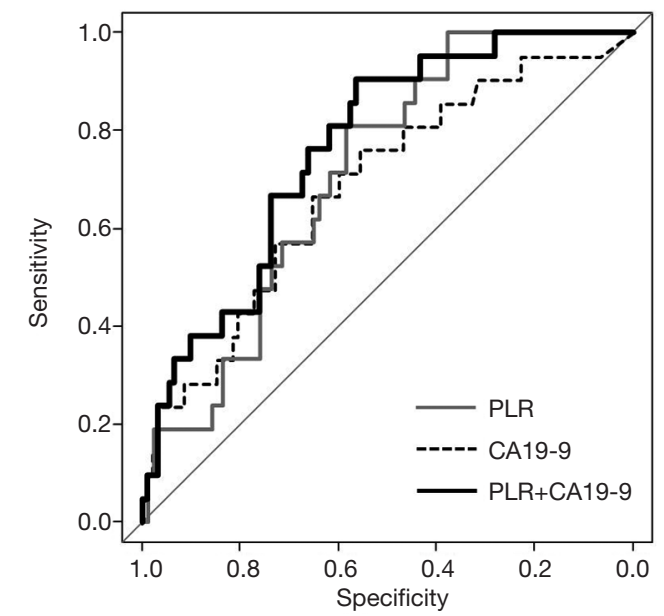

Figure 1 The receiver operating characteristic (ROC) curves of platelet-to-lymphocyte ratio (PLR), carbohydrate antigen (CA) 19-9, and the combination for predicting early recurrence. The area under the ROC curve (AUC) was 0.713 and 0.686 for PLR and CA19-9, respectively. The AUC increased to 0.765 for the combination of PLR and CA19-9. local recurrence was significantly less common in the ER group.

\section{Predictive ability of PLR, CA19-9, and the combination for $E R$}

The AUC, sensitivity, specificity, and positive and negative predictive values were calculated for PLR, CA19-9, and tumor size (Table 2). The AUCs were 0.713 (95\% CI: $0.608-0.818$ ), 0.686 (95\% CI: $0.558-0.814$ ), and 0.608 (95\% CI: 0.479-0.737) for PLR, CA19-9, and tumor size, respectively. When we selected PLR and CA19-9 and combined them for predictive ability analysis, the AUC increased to 0.765 (95\% CI: $0.664-0.866$ ), indicating that the combination has the greatest value for ER prediction (Table 2 and Figure 1).

\section{Multivariate analysis of risk factors for $E R$}

Table 3 shows the result of multivariate analysis performed 
Table 3 Multivariate logistic regression analysis of factors associated with early recurrence

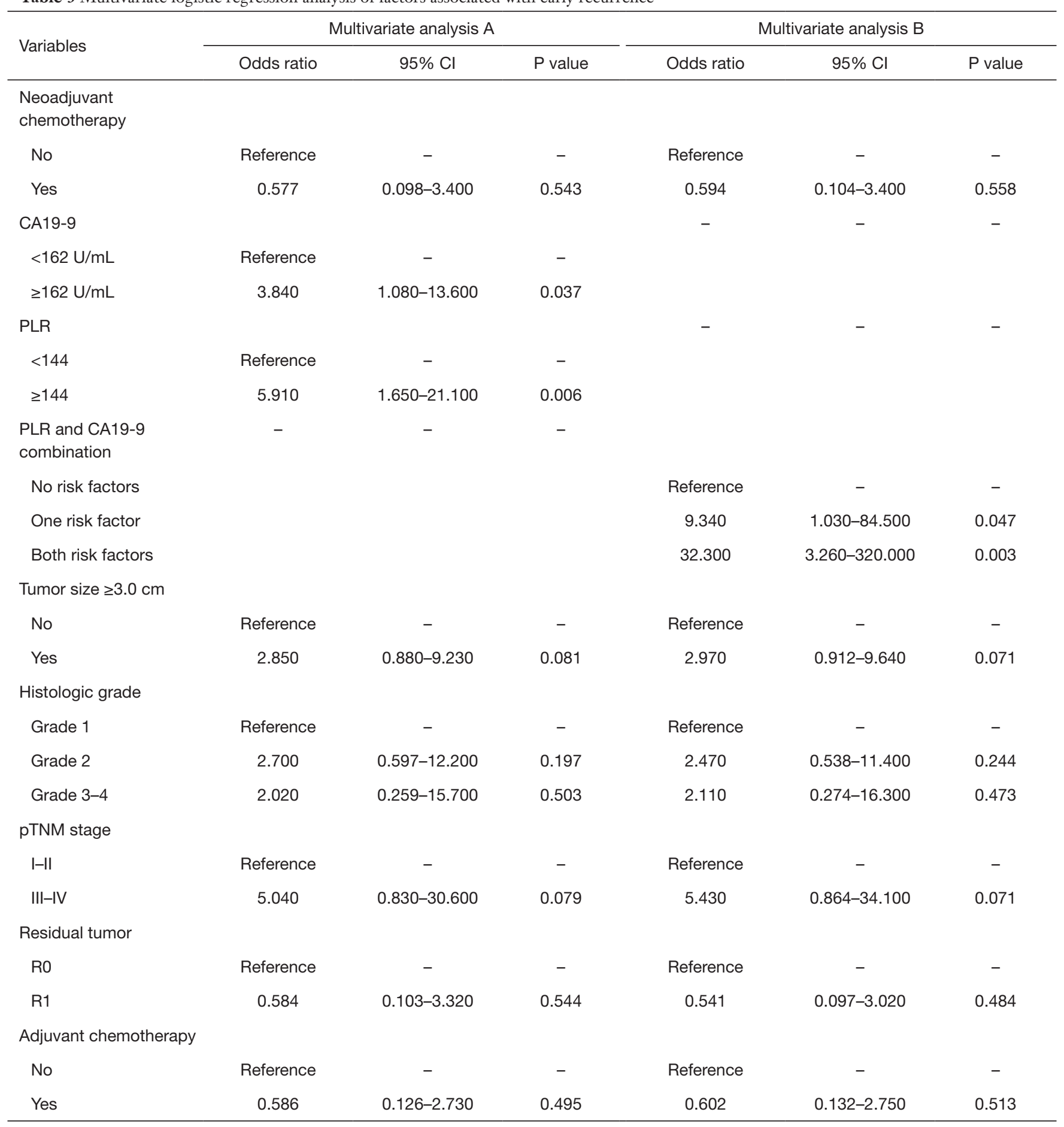

CA19-9, carbohydrate antigen 19-9; PLR, platelet-to-lymphocyte ratio. 
A

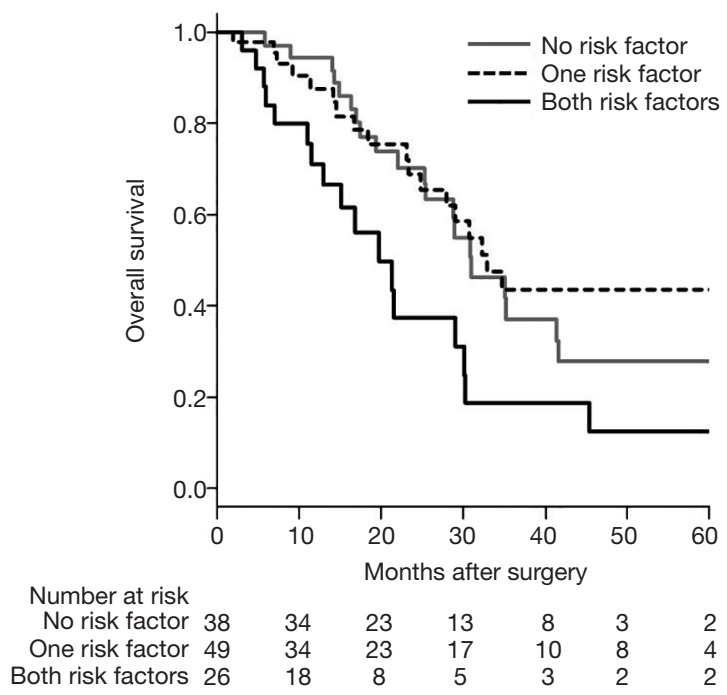

B

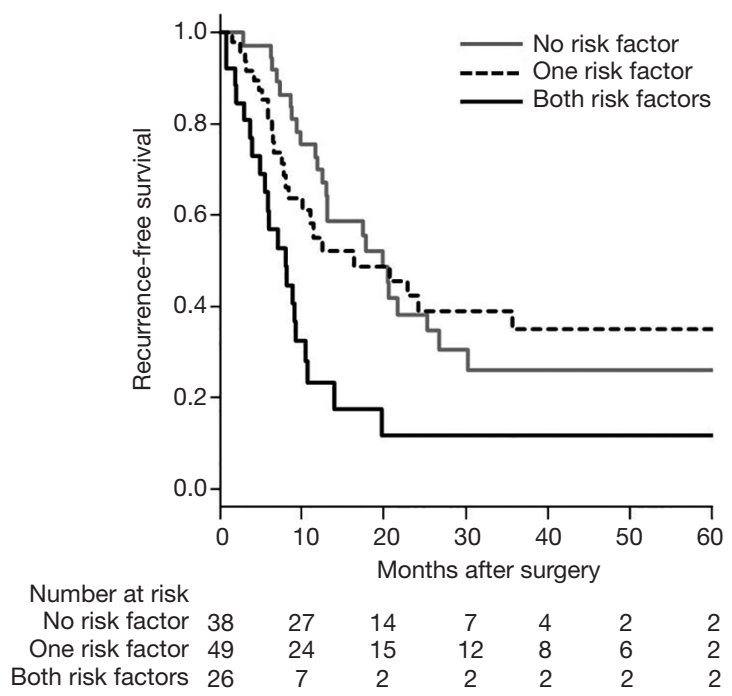

Figure 2 The overall (A) and recurrence-free (B) survival curves of patients with no risk factors [platelet-to-lymphocyte ratio (PLR) and carbohydrate antigen (CA) 19-9 both low; n=38], one risk factor (PLR and CA19-9 either high; n=49), and both risk factors (PLR and CA19-9 both high; $n=26$ ). Patients with both risk factors had significantly worse overall and recurrence-free survival compared to those with one or no risk factors.

to determine independent risk factors for ER. Because there were close associations between CA19-9 or PLR and their combination, they were separately analyzed in different multivariate analyses. In multivariate analysis A, CA19-9 $\geq 162 \mathrm{U} / \mathrm{mL}$ [odds ratio (OR), 3.840; 95\% CI: 1.080-13.600 $(\mathrm{P}=0.037)]$ and PLR $\geq 144$ [OR, 5.910; 95\% CI: 1.650-21.100 $(\mathrm{P}=0.006)]$ each independently affected $\mathrm{ER}$. When the subjects were classified into three subgroups based on the combination of low/high PLR and CA19-9 [no risk factors, PLR $<144$ and CA19-9 $<162 \mathrm{U} / \mathrm{mL}(\mathrm{n}=38)$; one risk factor, only PLR $\geq 144$ or CA19-9 $\geq 162 \mathrm{U} / \mathrm{mL}$ ( $\mathrm{n}=49$ ); both risk factors, PLR $\geq 144$ and CA19-9 $\geq 162 \mathrm{U} / \mathrm{mL}(\mathrm{n}=26)$ ], the classification was found to only be an independent predictor of $\mathrm{ER}$ in multivariate analysis $\mathrm{B}$. The OR relative to those with no risk factors was 9.340 (95\% CI: $1.030-84.500$ ) for those with one risk factor and 32.300 (95\% CI: 3.260$320.000)$ for those with both risk factors. Tumor size $\geq 3 \mathrm{~cm}$ was associated with ER in the univariate analysis but was not significant in both multivariate analyses A and B.

\section{Survival analysis}

The median OS in the ER group was 11.1 months, which was significantly shorter than that in the NER group [median, 32.9 months $(\mathrm{P}<0.001)$ ]. The median OS and RFS for patients with both risk factors were 19.8 and 8.0 months, respectively, which were significantly worse than those with one risk factor [median OS, 32.9 months $(\mathrm{P}=0.017)$; median RFS, 16.4 months $(\mathrm{P}=0.005)$ ] as well as for those with no risk factors [median OS, 31.0 months $(\mathrm{P}=0.030)$; median RFS, 19.9 months $(\mathrm{P}<0.001)$ ] (Figure $2 A, B)$. No significant differences in survival outcomes were found between patients with one risk factor and those with no risk factors.

\section{Effect of NAC against ER in BR-PDA patients}

To assess the effect of NAC against ER, the number of risk factors and rates of ER were compared between patients with BR-PDA receiving $(\mathrm{n}=13)$ and not receiving $(\mathrm{n}=11)$ NAC. As shown in Table 4, patients who received NAC were less likely to have risk factors for ER compared to those who did not $(\mathrm{P}=0.012)$. No patients who received NAC developed ER, but 4 of 11 patients who did not receive NAC experienced ER $(\mathrm{P}=0.031)$. Median OS was significantly prolonged in patients who received NAC compared to those who did not (32.9 vs. 14.9 months, $\mathrm{P}=0.011$ ).

\section{Discussion}

CA19-9 is an extensively studied biomarker for PDA and 
Table 4 Number of risk factors and rates of early recurrence in BR-PDA patients with or without neoadjuvant chemotherapy

\begin{tabular}{|c|c|c|c|c|c|c|c|c|c|}
\hline $\begin{array}{l}\text { Neoadjuvant } \\
\text { chemotherapy }\end{array}$ & $\mathrm{n}$ & \multicolumn{4}{|c|}{ Number of risk factors, $\mathrm{n}(\%)$} & \multicolumn{2}{|c|}{ ER } & \multicolumn{2}{|c|}{ OS, months } \\
\hline Yes & 13 & $8(61.5)$ & $4(30.8)$ & $1(7.7)$ & 0.012 & $0(0)$ & 0.031 & $32.9(22-N A)$ & 0.011 \\
\hline No & 11 & $5(45.5)$ & $0(0)$ & $6(54.5)$ & & $4(36.4)$ & & $14.9(4.7-19.8)$ & \\
\hline
\end{tabular}

BR-PDA, borderline resectable pancreatic ductal adenocarcinoma; ER, early recurrence; OS, overall survival; Cl, confidence interval; NA, not available.

provides information about resectability, prognosis, and therapeutic response (28-30). Its clinical utility to predict ER was demonstrated by several researchers and was reaffirmed by our study. For example, the cutoff value and AUC of CA19-9 for predicting ER were $228 \mathrm{U} / \mathrm{mL}$ and 0.683 by La Torre et al. and $529 \mathrm{U} / \mathrm{mL}$ and 0.6815 by Nishio et al., respectively $(13,15)$. Our cutoff CA19-9 value of $162 \mathrm{U} / \mathrm{mL}$ was lower, but the AUC of 0.686 was similar compared to their results, which suggests that CA19-9 predicts ER to some extent. However, according to a rough guide for classifying the accuracy of the diagnostic test, an AUC of 0.6-0.7 will only represent a fair (or not good) test (31). False positives are observed with CA19-9 in chronic pancreatitis and obstructive jaundice $(32,33)$, and $5-10 \%$ of patients can have a false negative because they do not produce CA19-9, which is common in patients with a Lewis phenotype Le $\mathrm{L}^{\mathrm{a}-\mathrm{b}-}$ (34). In fact, 7 patients $(6.2 \%)$ in our study cohort had a preoperative CA19-9 value of $0 \mathrm{U} / \mathrm{mL}$, but we included them in the analysis. Therefore, CA19-9 cannot always be used alone as a predictive risk factor of ER. CEA is another marker related with tumor burden of PDA and is often used to predict patient outcomes. However, the importance of CEA in ER prediction is unclear.

Inflammatory biomarkers in various malignancies have prognostic value. Zhou et al. performed a metaanalysis from 17 studies and found that high PLR was an unfavorable predictor of survival in PDA patients (35). High PLR was associated with shorter OS even in the subgroup analysis based on the adjusted potential confounders such as CA19-9, NLR, mGPS, tumor size, and nodal involvement. The mechanism by which high PLR affects poor survival of cancer patients remains unclear, but may be partly explained through an inflammatory process caused by cancer cells. The presence of tumor cells affects platelets and causes cancer-induced thrombosis (36). Platelets further release a number of growth factors, which support tumor growth, angiogenesis, and metastasis (36,37). Lymphocytes have a large role in cancer immune-surveillance, which prevents tumor development (38). Cancer-related inflammation causes suppression of antitumor immunity by recruitment of regulatory $\mathrm{T}$ cells and activation of chemokines, which results in tumor growth and metastasis (39). According to the results of this study, preoperative high PLR was a significant risk factor for ER, independent from the CA19-9 value. Furthermore, risk stratification based on a combination of PLR and CA19-9 predicted ER better than each marker alone. To our knowledge, this is the first study where PLR was combined with CA19-9 to improve the predictive ability of ER.

Large tumor size and mGPS-2 are correlated with ER $(13,14,40)$. Like PLR, mGPS is an index of systemic inflammation and is associated with poorer outcomes in cancer patients $(26,41)$. However, we did not find that mGPS significantly predicted ER, probably because of a small patient population in which only $4(3.5 \%)$ out of 113 patients had mGPS-2 preoperatively. ER was associated with tumor size $\geq 3 \mathrm{~cm}$ in the univariate analysis. We used pathological tumor size because it was occasionally difficult to estimate tumor size by a preoperative imaging examination. In an analysis of 64 patients who underwent Whipple procedures, Pahk et al. found that large tumor size by pathology, but not by CT or endoscopic US, was significantly correlated to ER (40). Thus, tumor size is a potential predictor of ER, but this factor can be accurately obtained postoperatively.

At present, there are no official guidelines for neoadjuvant therapy of PDA. However, intensive NAC or neoadjuvant chemoradiotherapy for BR-PDA has recently been adopted to achieve a more favorable prognosis. Jang et al. in the first prospective randomized controlled study published in 2018 demonstrated that gemcitabine-based chemoradiotherapy $(n=27)$ had an oncological benefit compared to that of upfront surgery $(\mathrm{n}=23)$ in terms of $\mathrm{R} 0$ resection $(51.8 \%$ vs. $26.1 \%, \mathrm{P}=0.004)$ and a 2 -year survival rate $(40.7 \%$ vs. $26.1 \%, \mathrm{P}=0.028)$ (42). In our study, distant metastasis was the dominant type of recurrence in ER 
patients. Furthermore, NAC for BR-PDA patients was associated with a reduced likelihood of having risk factors and with a low ER rate, which may lead to prolonged OS. These findings support the rationale for NAC to eliminate micrometastases or to select appropriate candidates for surgery.

There were several limitations in this study. First, this study was conducted at a single institution with a small sample size, which may explain the lack of survival difference between patients with one risk factor and those with no risk factors. Second, treatment strategies, including NAC regimens, were not uniform because of the retrospective nature of the study. Third, factors that may affect PLR value, such as patient liver function and withdrawal time of NAC before surgery, were not assessed. Fourth, patients who were only suspected of recurrence within 6 months (e.g., rises in CA19-9 without imaging evidence of recurrence) were assigned to the NER group. Finally, although there was an increase in predictive ability for ER when PLR was combined with CA19-9, the AUC was less than 0.8 and not sufficiently high.

In conclusion, we found that a combination of PLR and CA19-9 may help identify patients at a high risk of ER, which may improve management and therapeutic selection for patients with potentially R-PDA. NAC can be an effective alternative to upfront surgery to reduce the risk of ER for selective patients. However, our study was limited, and further well-designed large studies are needed to confirm the results.

\section{Acknowledgments}

None.

\section{Footnote}

Conflicts of Interest: The authors have no conflicts of interest to declare.

Ethical Statement: The authors are accountable for all aspects of the work in ensuring that questions related to the accuracy or integrity of any part of the work are appropriately investigated and resolved. This study was approved by the institutional ethics review board of Meiwa Hospital (No. No. H31-13) and was conducted in accordance with the Declaration of Helsinki.

\section{References}

1. Bray F, Ferlay J, Soerjomataram I, et al. Global cancer statistics 2018: GLOBOCAN estimates of incidence and mortality worldwide for 36 cancers in 185 countries. CA Cancer J Clin 2018;68:394-424.

2. Rahib L, Smith BD, Aizenberg R, et al. Projecting cancer incidence and deaths to 2030: the unexpected burden of thyroid, liver, and pancreas cancers in the United States. Cancer Res 2014;74:2913-21.

3. Brennan MF, Kattan MW, Klimstra D, et al. Prognostic nomogram for patients undergoing resection for adenocarcinoma of the pancreas. Ann Surg 2004;240:293-8.

4. Smeenk HG, Tran TC, Erdmann J, et al. Survival after surgical management of pancreatic adenocarcinoma: does curative and radical surgery truly exist? Langenbecks Arch Surg 2005;390:94-103.

5. Groot VP, Gemenetzis G, Blair AB, et al. Defining and Predicting Early Recurrence in 957 Patients With Resected Pancreatic Ductal Adenocarcinoma. Ann Surg 2019;269:1154-62.

6. Fischer R, Breidert M, Keck T, et al. Early recurrence of pancreatic cancer after resection and during adjuvant chemotherapy. Saudi J Gastroenterol 2012;18:118-21.

7. Von Hoff DD, Ervin T, Arena FP, et al. Increased survival in pancreatic cancer with nab-paclitaxel plus gemcitabine. N Engl J Med 2013;369:1691-703.

8. Conroy T, Desseigne F, Ychou M, et al. FOLFIRINOX versus gemcitabine for metastatic pancreatic cancer. $\mathrm{N}$ Engl J Med 2011;364:1817-25.

9. Ferrone CR, Marchegiani G, Hong TS, et al. Radiological and surgical implications of neoadjuvant treatment with FOLFIRINOX for locally advanced and borderline resectable pancreatic cancer. Ann Surg 2015;261:12-7.

10. Loehrer AP, Ferrone CR: Treatment of locally advanced pancreatic ductal adenocarcinoma. Dig Surg 2016;33:343-50.

11. Okada KI, Hirono S, Kawai M, et al. Phase I study of nabpaclitaxel plus gemcitabine as neoadjuvant therapy for borderline resectable pancreatic cancer. Anticancer Res 2017;37:853-8.

12. Paniccia A, Edil BH, Schulick RD, et al. Neoadjuvant FOLFIRINOX application in borderline resectable pancreatic adenocarcinoma: a retrospective cohort study. Medicine (Baltimore) 2014;93:e198.

13. La Torre $\mathrm{M}$, Nigri $\mathrm{G}$, Lo Conte $\mathrm{A}$, et al. Is a preoperative 
assessment of the early recurrence of pancreatic cancer possible after complete surgical resection? Gut Liver 2014;8:102-8.

14. Matsumoto I, Murakami Y, Shinzeki M, et al. Proposed preoperative risk factors for early recurrence in patients with resectable pancreatic ductal adenocarcinoma after surgical resection: A multi-center retrospective study. Pancreatology 2015;15:674-80.

15. Nishio K, Kimura K, Amano R, et al. Preoperative predictors for early recurrence of resectable pancreatic cancer. World J Surg Oncol 2017;15:16.

16. Shimizu T, Asakuma M, Tomioka A, et al. Span-1 and CA19-9 as Predictors of Early Recurrence and Lymph Node Metastasis for Patients with Invasive Pancreatic Cancer after Pancreatectomy. Am Surg 2018;84:109-13.

17. Kurahara H, Maemura K, Mataki Y, et al. A therapeutic strategy for resectable pancreatic cancer based on risk factors of early recurrence. Pancreas 2018;47:753-8.

18. Hsu CP, Lee LY, Hsu JT, et al. CD44 predicts early recurrence in pancreatic cancer patients undergoing radical surgery. In Vivo 2018;32:1533-40.

19. National Comprehensive Cancer Network. NCCN clinical practice guidelines in oncology. Version 2. Ft Washington, PA: NCCN, 2015.

20. Tsubamoto H, Sonoda T, Ikuta S, et al. Combination chemotherapy with itraconazole for treating metastatic pancreatic cancer in the second-line or additional setting. Anticancer Res 2015;35:4191-6.

21. Ikuta S, Sonoda T, Aihara T, et al. Results of Adjuvant Surgery after Combination Chemotherapy for Pancreatic Carcinoma with Multiple Liver Metastases. JOP 2015;16:469-74.

22. Kim J, Tang JY, Gong R, et al. Itraconazole, a commonly used antifungal that inhibits Hedgehog pathway activity and cancer growth. Cancer Cell 2010;17:388-99.

23. Takara K, Tanigawara Y, Komada F, et al. Cellular pharmacokinetic aspects of reversal effect of itraconazole on P-glycoprotein-mediated resistance of anticancer drugs. Biol Pharm Bull 1999;22:1355-9.

24. Chong $\mathrm{CR}, \mathrm{Xu} \mathrm{J}, \mathrm{Lu} \mathrm{J}$, et al. Inhibition of angiogenesis by the antifungal drug itraconazole. ACS Chem Biol 2007;2:263-70.

25. Sobin LH, Wittekind C. TNM Classification of Malignant Tumours. 7th edition. Oxford: Wiley-Blackwell, 2009:132-5.

26. Toiyama Y, Miki C, Inoue Y, et al. Evaluation of an inflammation-based prognostic score for the identification of patients requiring postoperative adjuvant chemotherapy for stage II colorectal cancer. Exp Ther
Med 2011;2:95-101.

27. Schemper M, Smith TL. A note on quantifying followup in studies of failure time. Control Clin Trials 1996;17:343-6.

28. Kiliç M, Göçmen E, Tez M, et al. Value of preoperative serum CA 19-9 levels in predicting resectability for pancreatic cancer. Can J Surg 2006;49:241-4.

29. Ferrone CR, Finkelstein DM, Thayer SP, et al. Perioperative CA19-9 levels can predict stage and survival in patients with resectable pancreatic adenocarcinoma. J Clin Oncol 2006;24:2897-902.

30. Ko AH, Hwang J, Venook AP, et al. Serum CA19-9 response as a surrogate for clinical outcome in patients receiving fixed-dose rate gemcitabine for advanced pancreatic cancer. Br J Cancer 2005;93:195-9.

31. Lee YY, Yang CK, Weng YM, et al. All Components of Metabolic Syndrome Are Associated with Microalbuminuria in a Chinese Population. PLoS One 2016;11:e0157303.

32. Furuya N, Kawa S, Hasebe O, et al. Comparative study of CA242 and CA19-9 in chronic pancreatitis. Br J Cancer 1996;73:372-6.

33. Marrelli D, Caruso S, Pedrazzani C, et al. CA19-9 serum levels in obstructive jaundice: clinical value in benign and malignant conditions. Am J Surg 2009;198:333-9.

34. Tempero MA, Uchida E, Takasaki H, et al. Relationship of carbohydrate antigen 19-9 and Lewis antigens in pancreatic cancer. Cancer Res 1987;47:5501-3.

35. Zhou Y, Cheng S, Fathy AH, et al. Prognostic value of platelet-to-lymphocyte ratio in pancreatic cancer: a comprehensive meta-analysis of 17 cohort studies. Onco Targets Ther 2018;11:1899-908.

36. Mezouar S, Frère C, Darbousset R, et al. Role of platelets in cancer and cancer-associated thrombosis: experimental and clinical evidences. Thromb Res 2016;139:65-76.

37. Goubran HA, Stakiw J, Radosevic M, et al. Platelets effects on tumor growth. Semin Oncol 2014;41:359-69.

38. Dunn GP, Old LJ, Schreiber RD. The immunobiology of cancer immunosurveillance and immunoediting. Immunity 2004;21:137-48.

39. Zhou X, Du Y, Huang Z, et al. Prognostic value of PLR in various cancers: a meta-analysis. PLoS One 2014;9:e101119.

40. Pahk E, Jesin R, Kluger Y, et al. Predictors of early recurrence of adenocarcinoma of the head of the pancreas after curative resection. JOP 2015;16:597-600.

41. McMillan DC. The systemic inflammation-based Glasgow Prognostic Score: a decade of experience in patients with 
cancer. Cancer Treat Rev 2013;39:534-40.

42. Jang JY, Han Y, Lee H, et al. Oncological benefits of neoadjuvant chemoradiation with gemcitabine versus upfront surgery in patients with borderline resectable pancreatic cancer: a prospective, randomized, open-label, multicenter phase 2/3 trial. Ann Surg 2018;268:215-22.

Cite this article as: Ikuta S, Sonoda T, Aihara T, Yamanaka N. A combination of platelet-to-lymphocyte ratio and carbohydrate antigen 19-9 predict early recurrence after resection of pancreatic ductal adenocarcinoma. Ann Transl Med 2019;7(18):461. doi: 10.21037/atm.2019.08.35 